\title{
On the scarcity of redshifted $\mathrm{OH}$ and millimetre-band molecular absorption
}

\author{
S. J. Curran ${ }^{\star}$ \\ School of Chemical and Physical Sciences, Victoria University of Wellington, PO Box 600, Wellington 6140, New Zealand
}

Accepted - Received —; in original form -

\begin{abstract}
Despite much searching, redshifted decimetre and millimetre-band absorption by molecular gas remains very rare, limited to just six systems at $z_{\mathrm{abs}} \approx 0.05$. Detection of these transitions can yield precise diagnostics of the conditions of the star forming gas in the earlier Universe, the hydroxyl $(\mathrm{OH})$ radical being of particular interest as in the $\lambda=18 \mathrm{~cm}$ ground state there are four different transitions located close to H I $21-\mathrm{cm}$ and thus detectable with the Square Kilometre Array and its pathfinders. The four transitions of $\mathrm{OH}$ have very different dependences on the fundamental constants, thus having much potential in testing for any evolution in these over large look-back times. By collating the photometry in a uniform manner, we confirm our previous hypothesis that the normalised $\mathrm{OH}$ absorption strength is correlated with the optical-near-infrared red colour of the sight-line. Applying this to the published searches, we find that all, but one (J0414+054), have simply not been searched sufficiently deeply. We suggest that this is due to the standard selection of sources with reliable optical redshifts introducing a bias against those with enough dust with which to shield the molecular gas. For the single source searched to sufficient depth, we have reason to suspect that the high degree of reddening arises from another system along the sight-line, thus not being inconsistent with our hypothesis. We also show that the same optical redshift bias can account for the scarcity of millimetre-band absorption.
\end{abstract}

Key words: galaxies: active - quasars: absorption lines - radio lines: galaxies - galaxies: high redshift - techniques: photometric - methods: data analysis

\section{INTRODUCTION}

Although neutral hydrogen (HI) has been detected through $\lambda=$ $21 \mathrm{~cm}$ absorption in 142 redshifted $\left(z_{\text {abs }} \gtrsim 0.1\right)$ sources (Curran 2021 and references therein), absorption by the hydroxyl $(\mathrm{OH})$ radical at $\lambda=18 \mathrm{~cm}$ remains rare, with only five detections at these redshifts (Chengalur et al. 1999; Kanekar \& Chengalur 2002; Kanekar et al. 2003, 2005). As well as tracing the molecular, and hence cool star-forming, gas, $\mathrm{OH}$ in this band is of particular interest as there are four hyperfine transitions (at 1612, 1665, 1667 \& $1720 \mathrm{MHz}$ ), each of which has a different dependence on various combinations of the fundamental constants (fine structure, electron-proton mass ratio, proton g-factor). The main advantage is that the absorption is known to arise along the same sight-line (Darling 2003), thus bypassing the possible line-of-sight differences which plague optical-band data (Murphy et al. 2003b) and could therefore contribute to the observed evolution in the fine structure constant over the past 12 billion years Webb et al. 2011 and references therein). Furthermore, the frequency shifts of the of radio-band lines are an order of magnitude more sensitive to

* Stephen.Curran@vuw.ac.nz a change in the fine structure constant than the optical-band lines (Curran et al. 2004).

Despite much searching, however, redshifted $\mathrm{OH}$ absorption remains scarce. As a possible explanation, Curran et al. (2006) noted that each of the detections arose along a very red sightline, with an optical-near-infrared colour of $V-K \gtrsim 5$, whereas the non-detections tended to be bluer. Although $\mathrm{H}_{2}$ has been detected in the rest-frame ultraviolet-band in $\approx 30$ damped Lyman- $\alpha$ absorption systems (DLAs, e.g. Levshakov \& Varshalovich 1985; Balashev et al. 2014; Noterdaeme et al. 2015), with colours $V-$ $K \lesssim 4$, these have much lower molecular fractions than detectable in $\mathrm{OH}$ (Curran et al. 2011a). This suggests that the selection of optically bright sources introduces a bias against dustier, and more molecular rich, sight-lines. Since then there have been many more searches for $\mathrm{OH}$ absorption (Curran et al. 2007, 2008, 2011b, 2016; Allison et al. 2017; Curran et al. 2017; Grasha et al. 2019, 2020; Dutta et al. 2020; Zheng et al. 2020; Gupta et al. 2021b), resulting in only one new detection (at $z_{\text {abs }}=0.05$, Gupta et al. 2018a).

Although we have previously noted a possible correlation between the $\mathrm{OH}$ absorption strength and the optical-near-infrared colour of the sight-line, this is rarely considered in the discussion of the low detection rates (e.g. Gupta et al. 2018a; Zheng et al. 2020). Given that surveys for decimetre-band absorption lines (H I \& OH) 
are currently underway on the pathfinder telescopes of the Square Kilometre Array (SKA), we revisit our hypothesis by adding the results of the $\mathrm{OH}$ searches made since. In order to be applicable to future large surveys, we formalise the collation of the magnitude measures, obtaining these in an automated and more uniform manner than previously. We then normalise the $\mathrm{OH}$ limits according to our suspicion that the line width is close to that of the H I $21-\mathrm{cm}$, before discussing the limits in the context of the detected $\mathrm{OH}$ and millimetre-band absorption systems.

\section{ANALYSIS}

\subsection{OH searches}

In the sixteen years since the last published $\mathrm{OH}$ detection, there has only been one further detection, at a redshift of $z_{\text {abs }}=0.05$ Gupta et al.2018a). Four of these six redshifted absorbers arise in galaxies intervening the sight-line to a more distant source providing the radio continuum, with the remaining two associated with the continuum source itself. Furthermore, for the $\mathrm{OH}$ absorbers we note that:

- All were previously detected in H I 21-cm absorption (Carilli et al. 1992, 1993, 1998; Chengalur et al. 1999; Kanekar \& Briggs 2003; Gupta et al. 2018a).

- At the time, at least five were located along very red sightlines, with optical-near-infrared colours of $V-K \gtrsim 5$, which suggested that the reddening was due to dust which protected the molecules from the ambient ultra-violet field (Curran et al. 2006).

- The same five also exhibit molecular absorption in the millimetre-bands Wiklind \& Combes 1994, 1995, 1996a b; Wiklind et al. 2018), four of which were detected in these transitions prior to the $\mathrm{OH}$ absorption and one prior to the $\mathrm{H}$ I absorption.

We therefore consider the $\mathrm{OH}$ searches to date in which $\mathrm{H}$ I $21-\mathrm{cm}$ absorption has been detected, which we list in Table 1

\subsection{Colours}

Previously (Curran et al. 2006; Curran et al. 2007, 2008, 2011b), the $V-K$ colours came from a variety of disparate sources in the literature. As well as being non-uniform, this is not ideal when considering a large number of sources, which will be the case in the analysis of SKA and SKA pathfinder data. We therefore use a similar method to that of Curran \& Moss 2019), where the photometry are scraped from NASA/IPAC Extragalactic Database (NED), the Wide-Field Infrared Survey Explorer (WISE, Wright et al. 2010) and the Two Micron All Sky Survey (2MASS, Skrutskie et al. 2006). If a datum fell into either the $V$ or $K$-bands, this was used, with multiple values being averaged. If not, this was extrapolated from a power-law fit to near-by bands - the WISE $W 1 \& W 2$-bands for $K$ and the $I, R \& B$-bands for $V$ (Fig. 1).

Our photometry scraping does not yield a $V$ magnitude for PKS 1830-21. Although, we had previously (e.g. Curran et al. 2007) used $V-K=6.25$, we have no record of the magnitude's

1 Specifically, the First Large Absorption Survey in H I on the Australian Square Kilometre Array Pathfinder (Allison et al. 2020) and the MeerKAT Absorption Line Survey (Gupta et al. 2021a)

${ }^{2}$ In the absence of an a priori redshift, spectral scans in the millimetre-band are very observationally expensive, due to the narrow relative bandwidths at $\nu \gtrsim 100 \mathrm{GHz}$ (Murphy et al. 2003a). origin. Being one of the few detections, this is important for our analysis and so on the presumption that $V>I=22.33$, and using the measured $K=15.38$, we assign $V-K>6.95$. We apply the same reasoning to the other sources with a $K$ magnitude but no $V$ measurement nor fit - for PKS $0428+20 R=20.0$, giving $V-K>5.5$, PKS 0500+019 $R=21.0$, giving $V-K>5.4$ and for PKS 1107-187 $R=19.6$, giving $V-K>4.4$. Although as yet unsearched for $\mathrm{OH}$ absorption, we note that PKS B1740-517, detected in CO $J=1 \rightarrow 2$ (Allison et al. 2019), thus being the only other detected redshifted rotational-band absorber (Sect. 3.3, has $R=20.9$ giving $V-K>5.6$.

\subsection{OH limits}

As seen from Table 1 the limits to the non-detections are given at a wide variety of spectral resolutions, therefore requiring normalisation (see Curran 2012). It was previously noted that for the $\mathrm{OH}$ detections the full width at half-maxima of the profiles were very similar to those of the $\mathrm{H} \mathrm{I}$ (Curran et al.2007) 3 Showing FWHM versus FWHM $_{\mathrm{H} \text { I }}$ in Fig. 2. we see this is also the case for the new detection, [HB89] 0248+430 (Gupta et al. 2018a), with the only real outlier being the $z_{\text {abs }}=0.247$ absorber in [HB89] $1413+135$. For this, $\mathrm{FWHM}_{\mathrm{H} \text { I }}=18 \mathrm{~km} \mathrm{~s}^{-1}$ (Carilli et al. 1992) compared to $\mathrm{FWHM}_{\mathrm{OH}} \approx 8 \mathrm{~km} \mathrm{~s}^{-1}$, which we obtain from our own fit as only the total width of $\approx 14 \mathrm{~km} \mathrm{~s}^{-1}$ is quoted Kanekar \& Chengalur 2002). With an rms noise level of $1.6 \mathrm{mJy}$ per $\Delta v=1.75 \mathrm{~km} \mathrm{~s}^{-1}$ and a peak of $7.8 \mathrm{mJy}$, from our fitting, this is a weak detection making the line width quite uncertain. Therefore, we have no reason to believe that $\mathrm{FWHM}_{\mathrm{OH}} \not \mathrm{FWHM}_{\mathrm{HI}}$. As per Curran et al. (2008, 2011b), we rescale the $\mathrm{OH}$ limits by $\sqrt{\mathrm{FWHM}_{\mathrm{HI}} / \Delta v}$, in order to give the $3 \sigma$ limit of a single channel "smoothed" to FWHM $_{\mathrm{OH}}$.

\subsection{Results}

Since our previous analysis of the relationship between the absorption strength and $V-K$ colour (Curran et al. 2011b), there has only been one new detection of $\mathrm{OH}$ (Gupta et al. 2018a), although a plethora of non-detections Curran et al. 2016, 2017; Gupta et al. 2018a, 2021b; Grasha et al. 2019, 2020; Dutta et al. 2020; Zheng et al. 2020). As stated above, all $\mathrm{OH}$ absorbers were previously detected in $\mathrm{HI} 21-\mathrm{cm}$ absorption and so we consider only these (Table 11. In order to incorporate the limits (Fig. 3), we use the Astronomy SURVival Analysis (ASURV) package (Isobe et al. 1986) which adds these as censored data points, allowing a generalised non-parametric Kendall-tau test 4 In Table 2. we summarise the two-sided probability of the observed distributions occuring by chance and the associated significance, assuming Gaussian statistics. Although, due to the small number of $\mathrm{OH}$ detections, the correlations are not greatly significant, it is apparent from Fig. 3 that all but one of $\mathrm{HI}$ absorbers searched for $\mathrm{OH}$ absorption have not reached the required sensitivity based upon the degree of reddening. For the only source which has been, $4 \mathrm{C}+05.19$ (J0414+054), there is much debate over the origin of its extremely red colour $(V-K=10.37)$, whether it arises in

\footnotetext{
3 As per the literature, when discussing $\mathrm{OH}$ we are referring to the $1667 \mathrm{MHz}^{2} \Pi_{3 / 2} J=3 / 2$ transition.

4 Since ASURV cannot apply an upper and lower limit simultaneously, we do not include the three non-detections with $V-K$ lower limits (Sect.2.2.
} 

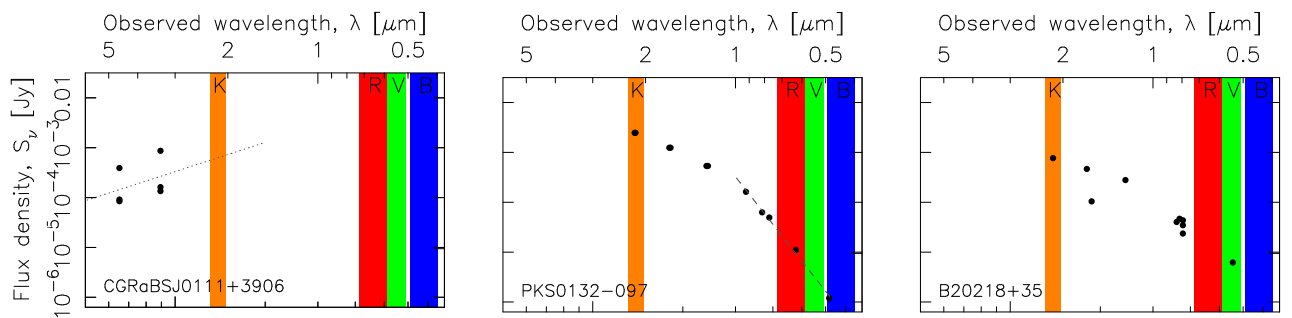

Observed wavelength, $\lambda[\mu \mathrm{m}]$
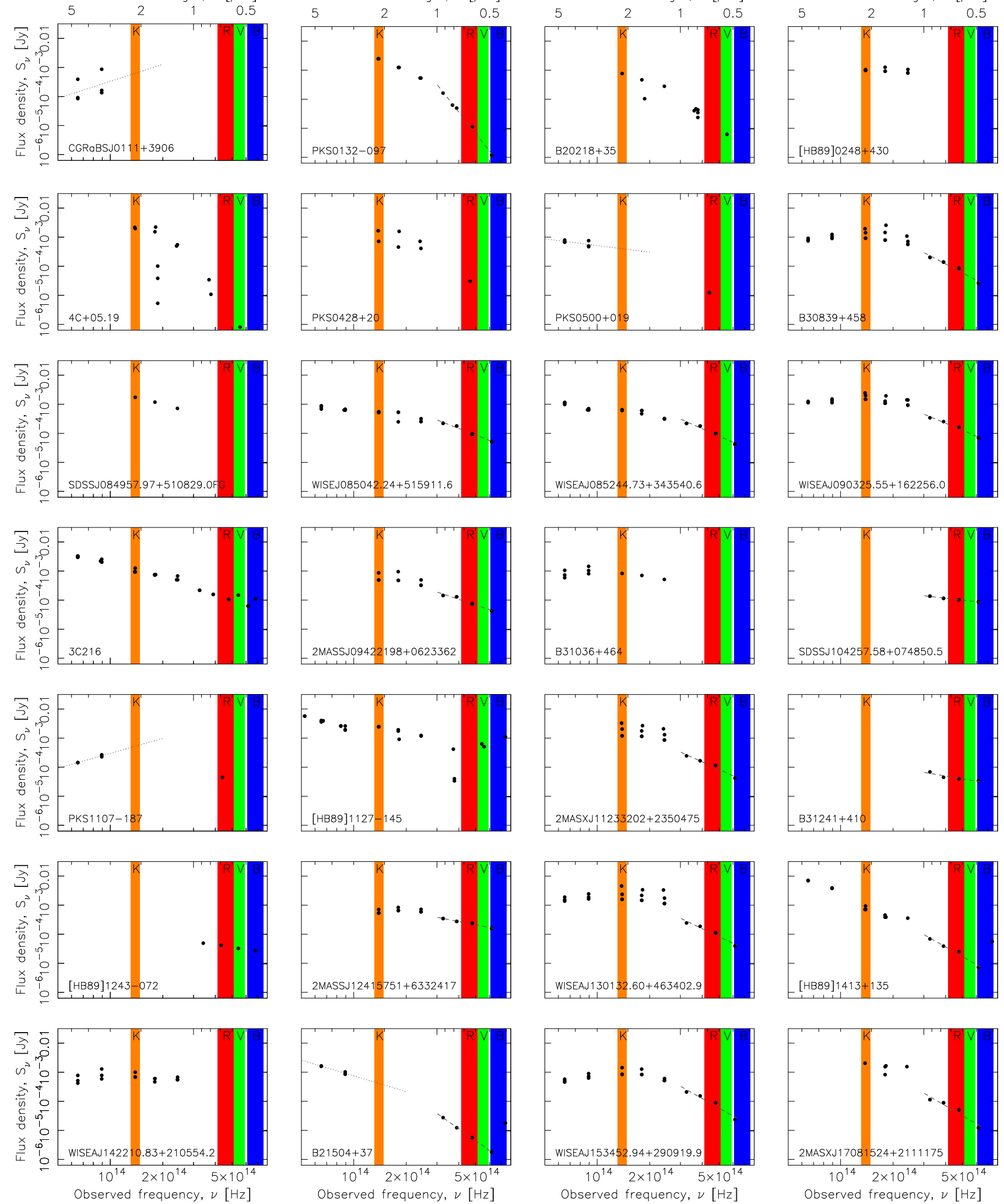

Figure 1. The near-infrared-visible photometry for the sight-lines searched in $\mathrm{OH}$ and detected in $\mathrm{H}$ I 21 -cm absorption. The coloured regions show the range of the $B, V, R$ and $K$-bands and the lines the least-square power-law fits from the neighbouring photometry, in the cases where the $V$ and $K$-bands are empty (dotted - near-infrared \& broken - optical). 
Table 1. The published OH $18-\mathrm{cm}$ searches for which H I $21-\mathrm{cm}$ absorption has been detected. The type (intervening or associated) is followed by the integrated optical depth $\left[\mathrm{km} \mathrm{s}^{-1}\right]$ and the full-width at half maximum of the profile $\left[\mathrm{km} \mathrm{s}^{-1}\right]$ for both the $\mathrm{H} \mathrm{I}$ and $1667 \mathrm{MHz} \mathrm{OH}$ absorption. Where $\mathrm{OH}$ is not detected, the spectral resolution which gives the quoted $3 \sigma$ upper limit is given. See Sect.2.2 for the $V$ and $K$ magnitudes.

\begin{tabular}{|c|c|c|c|c|c|c|c|c|c|c|}
\hline \multirow[t]{2}{*}{ Sight-line } & \multirow[t]{2}{*}{$z_{\mathrm{abs}}$} & \multirow[t]{2}{*}{ Type } & \multicolumn{3}{|c|}{ Hydrogen } & \multicolumn{3}{|c|}{ Hydroxyl } & \multirow[t]{2}{*}{$V$} & \multirow[t]{2}{*}{$K$} \\
\hline & & & $\int \tau_{\mathrm{H}_{\mathrm{I}}} d v$ & $\mathrm{FWHM}_{\mathrm{H} \text { I }}$ & Ref. & $\int \tau_{\mathrm{OH}} d v$ & $\mathrm{FWHM}_{\mathrm{OH}}, \Delta v$ & Ref. & & \\
\hline CGRaBS J0111+3906 & 0.66847 & A & 43.7 & 94 & O06 & $<0.84$ & 19 & $\mathrm{C} 10$ & - & 15.07 \\
\hline PKS 0132-097 & 0.76335 & I & 7.06 & 151 & K03a & 4.00 & 130 & K05 & 22.55 & 13.58 \\
\hline B2 $0218+35$ & 0.688466 & I & 2.65 & 53 & K03b & 0.401 & 61 & K03b & 21.92 & 14.85 \\
\hline [HB89] 0248+430 & 0.0519 & I & 0.42 & 21 & G18a & 0.08 & 19 & G18a & - & 14.56 \\
\hline $4 \mathrm{C}+05.19(\mathrm{~J} 0414+054)$ & 0.95974 & I & 0.88 & 49 & $\mathrm{C} 07$ & $<0.02$ & 4.3 & $\mathrm{C} 07$ & 24.14 & 13.77 \\
\hline PKS 0428+20 & 0.219 & A & 1.90 & 475 & V03 & $<0.10$ & 85.5 & O05 & $>20.0$ & 14.47 \\
\hline PKS 0500+019 & 0.58467 & A & 3.4 & 100 & C98 & $<0.23$ & 18 & $\mathrm{C} 06$ & $>21.0$ & 15.56 \\
\hline B3 $0839+458$ & 0.1919 & A & 25.9 & 79.2 & M17 & $<0.07$ & 1.64 & Z20 & 19.77 & 14.33 \\
\hline SDSS J084957.97+510829.0 & 0.3120 & I & 0.95 & 11 & G13 & $<0.091$ & 0.9 & G18a & - & 13.97 \\
\hline WISE J085042.24+515911.6 & 1.3265 & I & 15.3 & 30 & G09 & $<0.244$ & 1.1 & D20 & 19.17 & 15.26 \\
\hline WISEA J085244.73+343540.6 & 1.3095 & I & 6.91 & 48 & G09 & $<0.092$ & 0.4 & D20 & 19.40 & 15.08 \\
\hline WISEA J090325.55+162256.0 & 0.1823 & A & 8.98 & 163 & M17 & $<0.98$ & 1.62 & $\mathrm{Z} 20$ & 18.88 & 13.92 \\
\hline $3 \mathrm{C} 216(0906+430)$ & 0.670 & A & 0.67 & 285 & V03 & $<0.024$ & 30 & G19 & 18.48 & 14.56 \\
\hline 2MASS J09422198+0623362 & 0.12368 & A & 49.9 & 50 & S15 & $<0.070$ & 3.3 & G18a & 19.51 & 15.13 \\
\hline B3 1036+464 & 0.1861 & A & 14.75 & 86 & M17 & $<0.11$ & 1.63 & Z20 & - & 14.77 \\
\hline SDSS J104257.58+074850.5 & 0.0331 & I & 0.19 & 3.6 & B10 & $<0.027$ & 1.5 & G18a & 18.97 & - \\
\hline PKS 1107-187 & 0.48909 & A & 0.99 & 50 & $\mathrm{C} 10$ & $<0.077$ & 10 & $\mathrm{C} 10$ & $>19.6$ & 15.23 \\
\hline [HB89] 1127-145 & 0.31272 & I & 3.41 & 47 & G20 & $<0.098$ & 30 & G20 & 19.51 & 15.13 \\
\hline 2MASX J11233202+2350475 & 0.2070 & A & 5.01 & 220 & M17 & $<0.25$ & 1.66 & $\mathrm{Z} 20$ & 19.35 & 13.95 \\
\hline B3 $1241+410$ & 0.0169 & I & 2.24 & 15 & $\mathrm{G} 18 \mathrm{~b}$ & $<0.035$ & 1.4 & G18a & 20.01 & - \\
\hline [HB89] 1243-072 & 0.436734 & I & 1.50 & 4.4 & G20 & $<1.1$ & 30 & G20 & 20.13 & - \\
\hline 2MASS J12415751+6332417 & 0.143 & I & 2.90 & 40 & G10 & $<0.099$ & 1.2 & G18a & 18.20 & 15.14 \\
\hline WISEA J130132.60+463402.9 & 0.2055 & A & 4.24 & 172 & M17 & $<0.25$ & 1.65 & $\mathrm{Z} 20$ & 19.38 & 13.67 \\
\hline [HB89] 1413+135 & 0.24671 & A & 7.1 & 18 & C92 & 0.023 & 8 & K02 & 21.15 & 14.86 \\
\hline WISEA J142210.83+210554.2 & 0.1915 & $\mathrm{~A}$ & 8.36 & 180 & M17 & $<0.31$ & 1.64 & $\mathrm{Z} 20$ & - & 14.84 \\
\hline B2 $1504+37$ & 0.67343 & A & 24.5 & 57 & C98 & 0.42 & 45 & K02 & 22.68 & 15.52 \\
\hline WISEA J153452.94+290919.9 & 0.2010 & A & 6.77 & 234 & M17 & $<0.60$ & 1.65 & $\mathrm{Z} 20$ & 19.80 & 14.56 \\
\hline 2MASX J17081524+2111175 & 0.2241 & A & 28.4 & 211 & M17 & $<1.59$ & 1.68 & $\mathrm{Z} 20$ & 20.47 & 13.79 \\
\hline PKS 1830-21 & 0.885316 & I & 5.5 & 199 & C99 & 1.33 & 239 & C99 & $>22.3$ & 15.38 \\
\hline PKS 2052+005 & 0.20153 & I & 29.9 & 150 & D16 & $<0.03$ & 1.64 & $\mathrm{Z} 20$ & 21.13 & 15.43 \\
\hline PKS 2252-089 & 0.6064 & A & 11.0 & 94 & $\mathrm{C} 10$ & $<1.1$ & 10 & $\mathrm{C} 10$ & 22.30 & 16.91 \\
\hline $3 \mathrm{C} 459(2313+03)$ & 0.2199 & A & 0.388 & 164 & V03 & $<0.13$ & 30 & G19 & 17.44 & 13.92 \\
\hline
\end{tabular}

References: C92 - Carilli et al. (1992), C98 - Carilli et al. (1998), C99 - Chengalur et al. (1999), K02 - Kanekar \& Chengalur (2002), K03a Kanekar \& Briggs (2003), K03b - Kanekar et al. (2003), V03 - Vermeulen et al. (2003), K05 - Kanekar et al. (2005), O05 - O'Dea et al. (2005), O06 Orienti et al. (2006), C06-Curran et al. (2006), C07-Curran et al. (2007), G09-Gupta et al. (2009), B10 - Borthakur et al. (2010), C10 - Curran et al. (2011b), G10 - Gupta et al. (2010), G13 - Gupta et al. (2013), S15 - Srianand et al. (2015), D16 - Dutta et al. (2017), M17 - Maccagni et al. (2017), G18a - Gupta et al. (2018a), G18b - Gupta et al. (2018b), G19 - Grasha et al. (2019), D20 - Dutta et al. (2020), G20 - Grasha et al. (2020), Z20 Zheng et al. (2020).
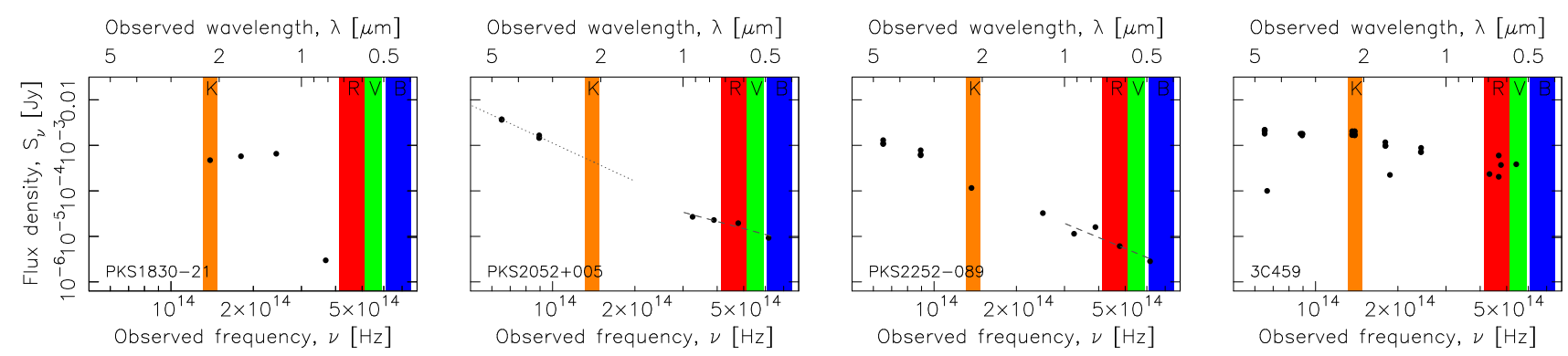

Figure 1. continued.

the intervening gravitational lens responsible for the H I absorption (Curran et al. 2007), the $z_{\mathrm{em}}=2.639$ background emitter (Falco et al. 1997; Tonry \& Kochanek 1999), which also exhibits strong H I 21-cm absorption (Moore et al. 1999), or another possible foreground object (Elíasdóttir et al. 2006; Tanna et al. 2013). We therefore flag the optical-near-infrared colour as $V-K<$
10.37. Using this upper limit and the $V-K$ lower limit to PKS 1830-21 (Sect. 2.2, results in a significance of $S(\tau)=$ $2.52 \sigma$ for the normalised $\mathrm{OH}$ line-strength and $2.76 \sigma$ for the unnormalised line-strength. These fall to $2.20 \& 2.37 \sigma$, respectively, when $V-K=10.37$ is used and $1.46 \& 2.05 \sigma$, respectively, for the five detections with $V-K$ measurements only. 


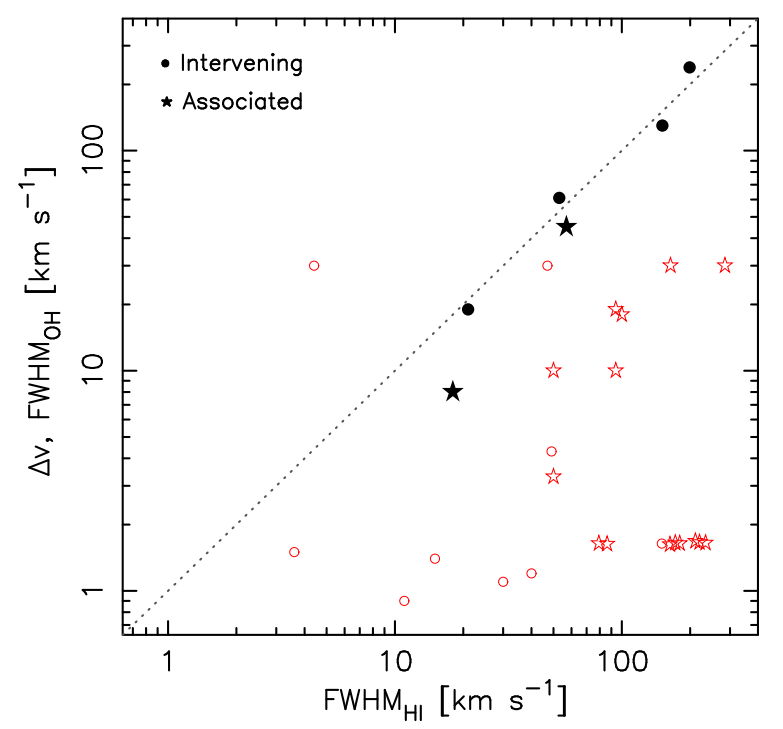

Figure 2. The full width at half-maximum of the $1667-\mathrm{MHz} \mathrm{OH}$ profile versus that of the $\mathrm{H} \mathrm{I} 21-\mathrm{cm}$ for the $\mathrm{OH}$ detected systems (filled symbols) and the quoted spectral resolution for the non-detections (unfilled). The dotted line shows $\mathrm{FWHM}_{\mathrm{OH}}=\mathrm{FWHM}_{\mathrm{HI}}$.

Table 2. The statistical significance of the correlations, $S(\tau)$, where $n$ is the sample size and $P(\tau)$ the probability of the distribution arising by chance.

\begin{tabular}{lccccc}
\hline & \multicolumn{3}{c}{ Normalised } & \multicolumn{2}{c}{ Un-normalised } \\
Description & $n$ & $P(\tau)$ & $S(\tau)$ & $P(\tau)$ & $S(\tau)$ \\
\hline With 4C $+05.19 V-K$ limit & 21 & 0.018 & $2.52 \sigma$ & 0.0058 & $2.76 \sigma$ \\
No 4C $+05.19 V-K$ limit & 21 & 0.028 & $2.20 \sigma$ & 0.018 & $2.37 \sigma$ \\
Detections only & 5 & 0.033 & $1.46 \sigma$ & 0.040 & $2.05 \sigma$ \\
\hline
\end{tabular}

In addition to $4 \mathrm{C}+05.19$, for one other source the normalised $\mathrm{OH}$ strength appears close to detection (Fig. 3 left) PKS 2052+005, which has $V-K=5.69$ and $\int \tau_{\mathrm{OH}} d v / \int \tau_{\mathrm{H}_{\mathrm{I}}} d v<$ 0.01. This limit is based upon the rms noise level of $\sigma_{\mathrm{rms}}=$ $0.6 \mathrm{mJy}$ per $\Delta v=1.65 \mathrm{~km} \mathrm{~s}^{-1}$ after removal of the bandpass ripple, although RFI still affects the bandpass close to the expected absorption frequency (Zheng et al. 2020).

In the bottom panels of Fig. 3 we show the binned values, where the upper limits are included via the Kaplan-Meier estimator Kaplan \& Meier 1958). This gives a maximum likelihood estimate based upon the parent population (Feigelson \& Nelson 1985) and requires at least one detection in each bin, limiting the number of bins to two. From this, it is clear that, even without the $V-K$ limit 5 , there is a positive correlation between the $\mathrm{OH}$ absorption strength and the degree of reddening.

\section{DISCUSSION}

\subsection{Depth of current $\mathrm{OH}$ searches}

By adding all of the published searched for $\mathrm{OH}$ absorption to date and formalising the measurement of the optical-near-infrared

\footnotetext{
${ }^{5}$ It is not possible to simultaneously include the $V-K$ limits in the binning, but we can see how the normalised $\mathrm{OH}$ absorption strength varies between the two $V-K$ bins.
}

colour, we confirm our previous assertion that the $\mathrm{OH}$ absorption strength is correlated with the $V-K$ colour (Curran et al. 2006). This has a physical motivation, since we expect higher molecular abundances along dustier sight-lines, which would be evident through a higher degree of reddening. Nevertheless, this is usually not a consideration when discussing the scarcity of redshifted $\mathrm{OH}$ absorption (e.g. Gupta et al. 2018a; Zheng et al. 2020). Here we show that, for the sensitivities reached by these surveys, the sources are simply not sufficiently red, which is most likely due to a bias towards the most optically bright sources, in which spectroscopic redshifts are more readily available (e.g. in DLAs). For instance, Gupta et al. (2018a) note that the absorber with the highest H I column density 6 in their sample, 2MASS J09422198+0623362, exhibits no $\mathrm{OH}$ absorption at a $3 \sigma$ limit of $\int \tau_{\mathrm{OH}} d v<0.07 \mathrm{~km} \mathrm{~s}^{-1}$ per $3.3 \mathrm{~km} \mathrm{~s}^{-1}$ channel. This has $V-K=4.38$ and, from the normalised fit (Fig. 3 left),

$$
\int \tau_{\mathrm{OH}} d v=\int \tau_{\mathrm{HI}} d v \times 10^{0.671(V-K)-6.078}
$$

where $\int \tau_{\mathrm{H}_{\mathrm{I}}} d v=49.9 \mathrm{~km} \mathrm{~s}^{-1}$, we expect $\int \tau_{\mathrm{OH}} d v=$ $0.036 \mathrm{~km} \mathrm{~s}^{-1}$ and, from the un-normalised fit (Fig. 3 right),

$$
\int \tau_{\mathrm{OH}} d v=10^{0.685(V-K)-5.328},
$$

we expect $\int \tau_{\mathrm{OH}} d v=0.005 \mathrm{~km} \mathrm{~s}^{-1}$. Resampling the $3 \sigma$ limit to FWHM $_{\mathrm{HI}}$, gives $\int \tau_{\mathrm{OH}} d v<0.3 \mathrm{~km} \mathrm{~s}^{-1}$ per $\Delta v=50 \mathrm{~km} \mathrm{~s}^{-1}$, which is significantly higher than either estimate thus explaining the non-detection.

For the only $\mathrm{H}$ I absorber which has been searched sufficiently deeply, but not detected in $\mathrm{OH}$, there has been some debate over the source of the reddening, whether in the $z_{\mathrm{abs}}=0.96$ gravitational lens, the $z_{\mathrm{em}}=2.64$ host galaxy, or an another intervening galaxy (Sect.2.3. We note that the H I 21-cm absorption in the host galaxy is five times stronger than in the lens $\left(\int \tau_{\mathrm{H}_{\mathrm{I}}} d v=4.2 \mathrm{~km} \mathrm{~s}^{-1}\right.$, Moore et al. 1999, cf. $0.9 \mathrm{~km} \mathrm{~s}^{-1}$, Curran et al. 2007) and, since the 21-cm absorption strength is also correlated with $V-K$ Curran et al. 2019), we may expect the bulk of the reddening to occur in the host galaxy. However, Moore et al. (1999) did not detect $\mathrm{HCN} J=0 \rightarrow 1$ absorption to a $3 \sigma$ optical depth limit of $\int \tau_{\mathrm{HCN}} d v<0.05 \mathrm{~km} \mathrm{~s}^{-1}$ at $\Delta v=19 \mathrm{~km} \mathrm{~s}^{-1}$. It should be noted, however, that, although there is strong $\mathrm{H} \mathrm{I}$ and $\mathrm{OH}$ absorption in the $z_{\text {abs }}=0.76$ absorber towards PKS 0132-097, $\mathrm{HCO}^{+} J=1 \rightarrow 2$ was undetected at a $3 \sigma$ limit of $\int \tau_{\mathrm{HCO}^{+}} d v<0.03 \mathrm{~km} \mathrm{~s}^{-1}$ per $5 \mathrm{~km} \mathrm{~s}^{-1}$ channel (Kanekar et al. 2005). This was subsequently detected with $\int \tau_{\mathrm{HCO}^{+}} d v=42 \mathrm{~km} \mathrm{~s}^{-1}$ (Wiklind et al. 2018) and so it is possible that $4 \mathrm{C}+05.19$ could also be subject to the same apparent variability. In any case, the non-detection of $\mathrm{HCN}$ could be due to the smaller filling factor of the millimetre-band molecular absorption (Zwaan \& Prochaska 2006), whereas the $\mathrm{OH}$ is more diffuse, as evident through the profile widths tracing those of the H I (Fig. 2).

\subsection{Reddening of $\mathbf{4 C}+\mathbf{0 5 . 1 9}$}

In order to check the validity of placing an upper limit on the red colour due to the $z_{\text {abs }}=0.96$ gravitational lens towards $4 \mathrm{C}+05.19$, we can compare this sight-line with a large sample of quasi-stellar objects (QSOs) to gauge just how likely it is that the reddening

${ }^{6}$ Actually integrated optical depth (see Curran et al. 2013). 

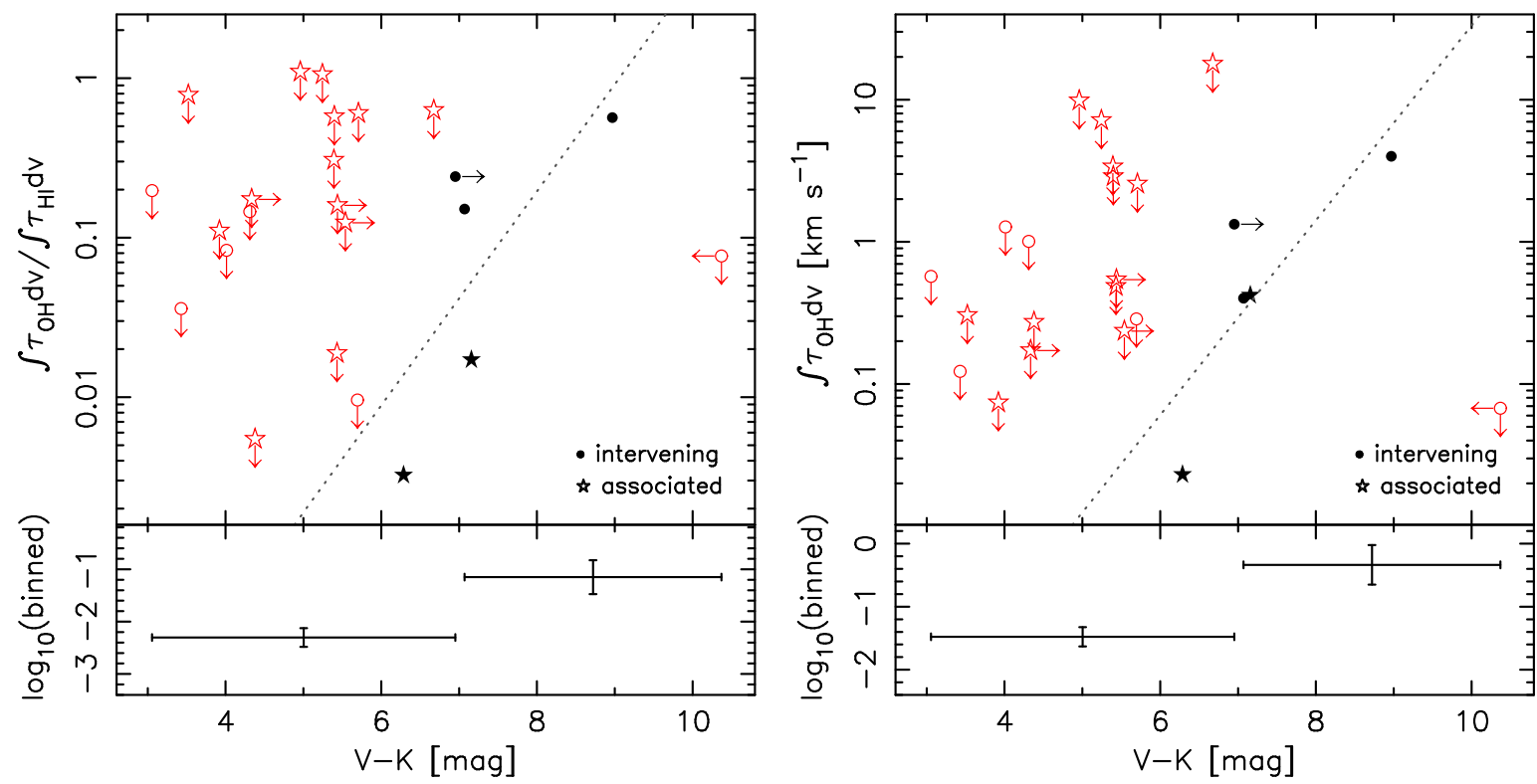

Figure 3. The normalised (left) and un-normalised (right) $\mathrm{OH}$ line strengths for the detections (filled symbols) and the $3 \sigma$ upper limits for the non-detections (unfilled). The dotted line shows the least-squares power-law fit to the five detections ([HB89] 0248+430 is missing since it has no optical-band photometry, Fig.11, incorporating the lower limit to the colour. The binned values show the upper limit to the mean ratio and the mean ratio $\pm 1 \sigma$.

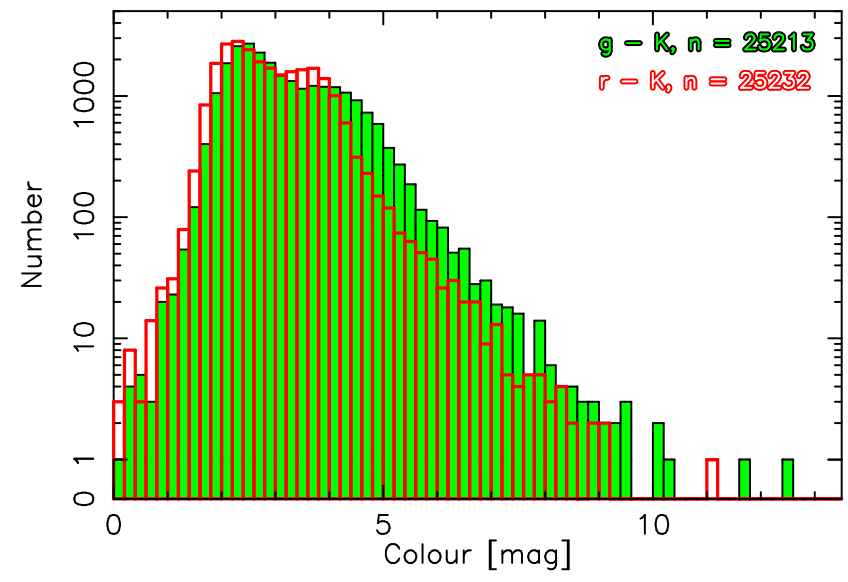

Figure 4. The $g-K$ (filled histogram) and $r-K$ (unfilled histogram) colours available for the 100337 SDSS DR12 QSOs for which we have the photometry.

is due to a single object. For this we use the photometry compiled by Curran et al. (2021) for the first 100337 QSOs with accurate spectroscopic redshifts $(\delta z / z<0.01)$ of the Sloan Digital Sky Survey (SDSS) Data Release 12 (DR12, Alam et al. 2015). With a central wavelength of $\lambda=551 \mathrm{~nm}$, the $V$-band is located between the SDSS $r(\lambda=623 \mathrm{~nm})$ and $g(\lambda=477 \mathrm{~nm})$ bands. We would therefore expect $r-K \lesssim 10.37 \lesssim g-K$ for 4C +05.19. Only two sources satisfy this condition (Fig. 4), SDSS $\mathrm{J} 113415.20+392826.1\left(z_{\mathrm{em}}=4.824\right)$, with $g-K=12.56$, and SDSS J150759.72+041512.1 $\left(z_{\mathrm{em}}=1.703\right)$, with $g-K=11.73$ (and $r-K=11.19$ ). Out of $\approx 25000$ sources, this makes the colour of $4 \mathrm{C}+05.19$ extremely rare. We note that the two SDSS sources are also at high redshift, thus being more likely to be subject to additional reddening due to intervening gas along the sight- line 7 It is therefore perfectly plausible that all of the reddening towards $4 \mathrm{C}+05.19$ is not solely due to the intervening lens and that the host galaxy would be a good candidate for $\mathrm{OH}$ absorption. Assuming the power-law fits to the detected $\mathrm{OH}$ absorbers, we estimate the reddening due to the $z_{\text {abs }}=0.96$ gravitational lens to be $V-K \leqslant 7.4$ (Equ. 1) and $V-K \leqslant 6.1$ (Equ. 2), both of which remain relatively high limits.

\subsection{Millimetre-band absorption}

In the millimetre ( $\mathrm{mm}$ )-band the many rotational transitions of the large variety of molecules provide an excellent probe of the physical and chemical conditions of the gas and its potential for star formation (e.g. Israel 1988; Aalto et al. 1995; Henkel et al. 2005). Sensitive temperature measurements from the relative line strengths can also offer extremely accurate measures of the cosmic evolution of the microwave background temperature at various look-back times (Wiklind \& Combes 1997; Muller et al. 2013) and time delays due to molecular absorbing gravitational lenses can be used to measure cosmological parameters (Wiklind \& Combes 2001). Furthermore, since these transitions are insensitive to the value of the fine structure constant, but sensitive to the electronproton mass ratio, mm-band lines provide useful "anchors" to measure shifts in the line frequencies and diagnostics of the values of the fundamental constants at large look-back times (Drinkwater et al. 1998; Curran et al. 2004).

However, these are equally as rare, with the five $\mathrm{OH}$ absorbers, in conjunction with the $\mathrm{CO} J=1 \rightarrow 2$ absorption in the $z=0.44$ radio galaxy PKS B1740-517 Allison et al. 2019), constituting all of the redshifted mm-band absorbers. There

7 The obvious way to quantify this would be the correlation between colour and redshift, but since this is in the observed-frame it is an inconsistent measure of the source properties over a large redshift range Curran \& Moss 2019). 
has also been a number of non-detections of the strongest transitions $\left(\mathrm{CO}, \mathrm{HCO}^{+} \& \mathrm{HCN} 8\right.$ published (Wiklind \& Combes 1995, 1996b; Drinkwater et al. 1996; Murphy et al. 2003a; Curran et al. 2008, 2011a), the majority of which have been searched to sufficiently deep sensitivities based upon their $V-K$ colour Curran et al. 2011a). However, applying the same prerequisite as for the OH searches, only three, [HB89] 0438-436, PKS 0500+019 \& PKS 1107-187, have been detected in H I 21-cm absorption 10 The latter two belong to the $\mathrm{OH}$ sample (Table 1 and have been searched in $\mathrm{HCO}^{+} 1 \rightarrow 2$ and $\mathrm{CO} 0 \rightarrow 1$, respectively Curran et al.2011a), with [HB89] 0438-436 having been searched in CO $2 \rightarrow 3$ (Drinkwater et al. 1996).

Again, the noise levels of the non-detections are quoted at various spectral resolutions $\left(0.51-3.4 \mathrm{~km} \mathrm{~s}^{-1}\right)$ and so we must normalise each one according to the expected line-width. In Fig. 5] we show the approximate millimetre band profile widths 11 versus that of the H I $21-\mathrm{cm}$. From this we see that, in general, $\mathrm{FWHM}_{\mathrm{mm}} \not$ FWHM $_{\mathrm{HI}}$, with the divergence decreasing with increasing line width. Although we fit the data with a power law, we suspect that the $\mathrm{HI}$ and $\mathrm{mm}$ line widths remain equal at $\mathrm{FWHM}_{\mathrm{HI}} \gtrsim$ $100 \mathrm{~km} \mathrm{~s}^{-1}$. This non-equivalence could be due to the dense mm-band absorbing gas being more localised than a more diffuse $\mathrm{OH}$, although this requires the $\mathrm{OH}$ to be non-coincident with the other molecular species. Alternatively, Curran et al. (2007) suggested that the non-detection of mm-band absorption in $4 \mathrm{C}+05.19$ (and PKS 0132-097) could be due to the millimetre wavelength emission, as opposed to or in addition to the absorption, being much more localised than that at decimetre wavelengths, so that a molecular cloud has a much smaller chance of occulting the higher frequency radiation. However, as seen in Fig. 5 $\mathrm{FWHM}_{\mathrm{mm}} \rightarrow$ $\mathrm{FWHM}_{\mathrm{H} \text { I }}$ with increasing line width, which would suggest that these have correspondingly larger mm-emission regions. While high resolution imaging in the millimetre-band is available for the two broadest absorbers, PKS 0132-097 and PKS 1830-21, revealing the lensed components (Wiklind et al.2018; Muller et al. 2020, respectively), imaging of the remaining sight-lines would be required to confirm this.

Resampling the limits to the FWHM expected from the $\mathrm{HCO}^{+}$ $1 \rightarrow 2$ transition 12 in Fig. 6, we show the limits together with the absorption strengths of the most commonly detected transitions versus the optical-near-infrared colour. Note that some of the detections are lower limits, since the millimetre-band absorption is often optically thick, and so the assumption that the optical depth, $\tau$, is the ratio of the observed optical depth $\tau_{\text {obs }}$ to the fraction of flux intercepted, $f$, cannot be made. That is,

$$
\tau \equiv-\ln \left(1-\frac{\tau_{\text {obs }}}{f}\right) \not \approx \frac{\tau_{\text {obs }}}{f} .
$$

Due to the lower limits, we cannot obtain a least-squares fit and, although the correlations are weak due to the very limited numbers, an increase in $\int \tau_{\mathrm{mm}} d v$ (and $\int \tau_{\mathrm{mm}} d v / \int \tau_{\mathrm{H}} d v$ ) with $V-K$

8 See Combes \& Wiklind (1997); Muller et al. 2011, 2016, 2017); Wallström et al. (2019); Tercero et al. (2020) for the other detected transitions.

957 transitions along 43 different sight-lines.

10 Although several are DLAs, i.e. strongly detected in H I Lyman- $\alpha$ absorption.

11 Often these are not given Wiklind \& Combes 1998, 1996a; Wiklind et al. 2018; Muller et al. 2013) and have to be estimated from $\mathrm{FWHM} \approx \int \tau d v / \tau_{\text {peak }}$ (e.g. Allison et al. 2013).

12 The CO data could not be fit and the smaller sample of HCN data have a similar fit. is evident. From this it is clear that, although many sources have been searched to sufficiently deep limits on the basis of their colour alone Curran et al. 2011a), those few which have also been detected in H I 21-cm fall far short of the required sensitivities. This re-emphasises the need to target extremely red sight-lines $(V-K \gtrsim$ 6 ), which have previously been detected in $21-\mathrm{cm}$ absorption.

\section{CONCLUSIONS}

Since the suggestion that the strength of redshifted $\mathrm{OH}$ absorption is correlated with the optical-near-infrared colour of the sight-line (Curran et al. 2006), there have been many unsuccessful searches for $\mathrm{OH}$ absorption. However, these studies do not take the red colour into account, leading to some puzzlement with regard to the continual non-detections (e.g. Gupta et al.2018a). Here we add the new to the previous searches and, taking into account that all of the six known $\mathrm{OH}$ absorbers were previously detected in $\mathrm{H}$ I $21-\mathrm{cm}$ absorption:

(i) In order to be applicable to future searches with the SKA and its pathfinders, rather than compiling the magnitudes from the variety of disparate sources in the literature (e.g. Curran et al. 2008), we automate and formalise the attainment of the $V-K$ colour by:

(a) Scraping the photometry from NED, 2MASS and WISE, extracting the $V$ and $K$ magnitudes from the former two.

(b) Where these are not available, extrapolating from near-by bands (WISE $W 1 \& W 2$ for the mid-infrared and $R \& B$ for the visible).

(c) And, where only a single optical-band photometry datum exists which is outside, but close to, the $V$-band, we use this to place a limit on the $V$ magnitude.

(ii) In order to normalise the limits, since FWHM $_{\mathrm{OH}} \approx$ FWHM $_{\mathrm{H}}$, we re-sample the limits to $\mathrm{FWHM}_{\mathrm{H}}$, giving the $3 \sigma$ limit of a single channel smoothed to $\mathrm{FWHM}_{\mathrm{OH}}$.

From the resulting $\mathrm{OH}$ absorption strengths we find that, according to their $V-K$ colour, only one of the published limits is sufficiently sensitive to detect $\mathrm{OH}$ absorption. This is in the $z_{\mathrm{abs}}=0.96$ gravitational lens towards $4 \mathrm{C}+05.19$ (Curran et al. 2007). However, this sight-line contains at least one other H I 21-cm absorber (Moore et al. 1999) and the source of its extremely red colour $(V-K=10.37)$ has long been a subject of debate (Falco et al. 1997; Tonry \& Kochanek 1999; ; Elíasdóttir et al.2006). Comparing the colour with those of a large sample of QSOs, we find this to be at the tail of the $V-K$ distribution $\left(\approx 8 \times 10^{-5}\right.$ of the sample). Using the $V-K$ fits to the known $\mathrm{OH}$ absorbers, we estimate the colour due to the lens to be $V-K \leqslant 6.1$ (based upon the unnormalised $\int \tau_{\mathrm{OH}} d v$ limit $)$ and $V-K \leqslant 7.4\left(\int \tau_{\mathrm{OH}} d v / \int \tau_{\mathrm{H}_{\mathrm{I}}} d v\right.$ limit). Given that the $21-\mathrm{cm}$ absorption in the $z_{\mathrm{em}}=2.639$ continuum source is five times stronger than in the lens, suggests that the host quasar is likely to contribute significantly to the degree of reddening.

Using $V-K<10.37$ for $4 \mathrm{C}+05.19$ and adding the limits as censored data points, we find that the observed $\int \tau_{\mathrm{H}} d v / \int \tau_{\mathrm{OH}} d v-V-K$ and $\int \tau_{\mathrm{OH}} d v-V-K$ distributions to be significant at $S(\tau)=2.52 \sigma$ and $2.76 \sigma$, respectively. Although not highly significant, due to the low number of non-censored data points (five of the detections), binning of the data shows a clear correlation between the normalised $\mathrm{OH}$ strength and the $V-K$ colour of the sight-line, thus confirming that the degree of reddening is a key contributor in the detection of molecular absorption. 

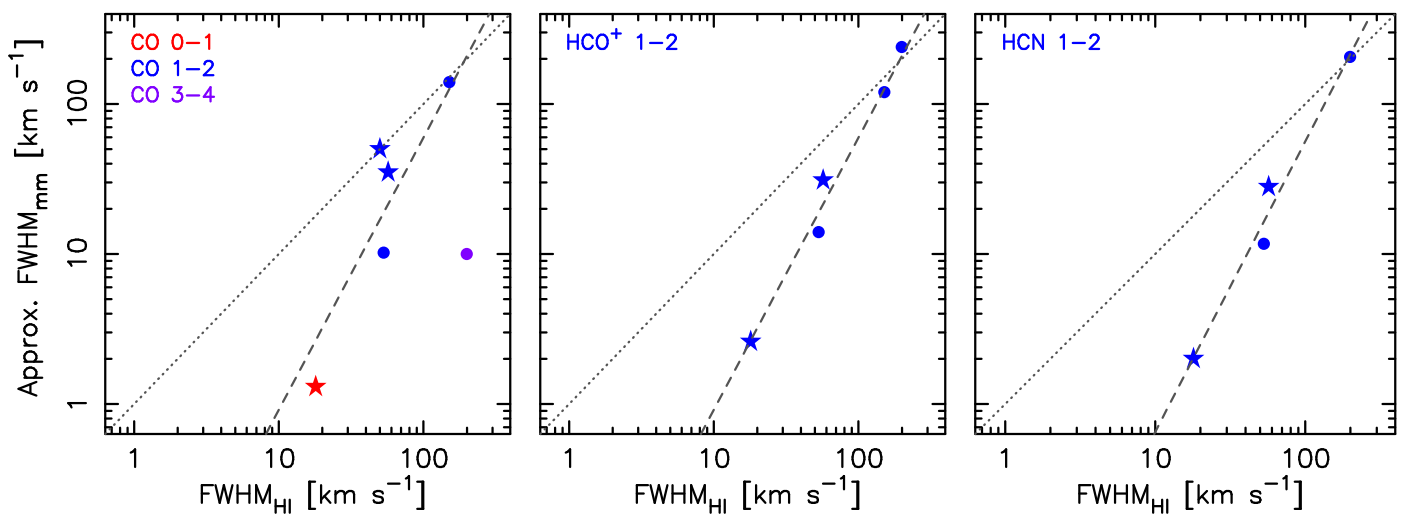

Figure 5. The full width at half-maximum of the $\mathrm{CO}$ (left), $\mathrm{HCO}^{+}$(middle) and $\mathrm{HCN}$ (right) versus that of the $\mathrm{H}$ I 21 -cm for the millimetre-band detected systems. The dotted line shows $\mathrm{FWHM}_{\mathrm{mm}}=\mathrm{FWHM}_{\mathrm{HI}}$ and the dashed lines the least-squares fit to the $\mathrm{HCO}^{+}$(which is also used in the left panel) and $\mathrm{HCN}$ $1 \rightarrow 2$ transitions

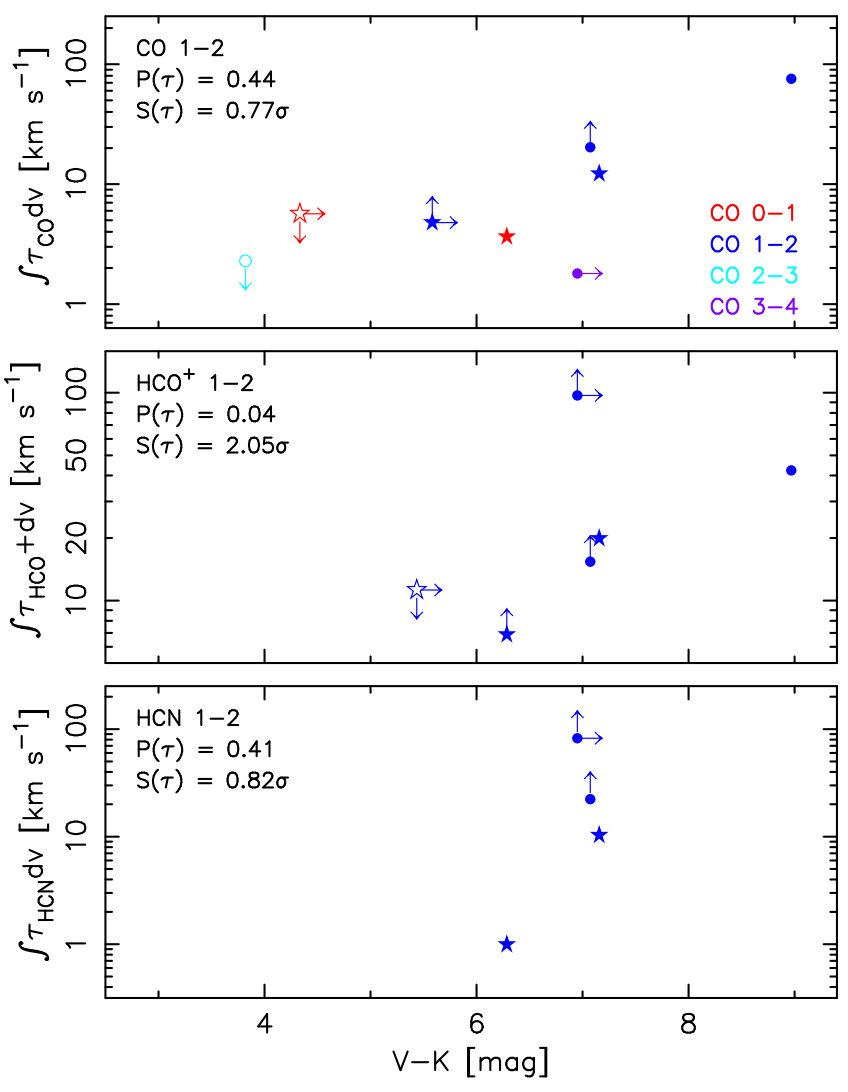

Figure 6. The $\mathrm{CO}$ (top), $\mathrm{HCO}^{+}$(middle) and $\mathrm{HCN}$ (bottom) line strengths for the rotational-band absorbers. The statistics are shown for the $J=$ $1 \rightarrow 2$ detections (filled symbols). Note the relative weakness of the $\mathrm{CO}$ $3 \rightarrow 4$ transition, indicating the low excitation temperature $\left(T_{\mathrm{ex}} \sim 10 \mathrm{~K}\right.$, Wiklind \& Combes 1998).

Since five of the redshifted $\mathrm{OH}$ absorbers are the same sources in which the equally elusive mm-band absorbers have been detected, we apply the same prerequisite of $\mathrm{HI} 21-\mathrm{cm}$ absorption. Although several sources have been searched in $\mathrm{CO}$ and $\mathrm{HCO}^{+}$ absorption to sufficiently deep limits based upon their $V-K$ colour (Curran et al. 2011a), those three which have been detected in H I 21-cm have not. Thus, we demonstrate that the degree of reddening is also crucial in the detection of the rotational transitions. This could explain the severe dearth in redshifted mm-band absorption (also limited to six detections), with previous searches having been biased towards sources which are sufficiently bright in the optical-bands (e.g. damped Lyman- $\alpha$ absorbers) to yield a redshift, to which to tune the narrow band millimetre receivers. As per the $\mathrm{OH}$, we therefore suggest that sight-lines with $V-K \gtrsim 6$ detected in $21-\mathrm{cm}$ absorption should be targetted in order to increase the number of known molecular absorbers in both the millimetre and decimetre-bands.

\section{ACKNOWLEDGEMENTS}

I wish to thank the referee for their helpful comments. This research has made use of the NASA/IPAC Extragalactic Database (NED) which is operated by the Jet Propulsion Laboratory, California Institute of Technology, under contract with the National Aeronautics and Space Administration and NASA's Astrophysics Data System Bibliographic Service. This research has also made use of NASA's Astrophysics Data System Bibliographic Service and ASURV Rev 1.2 (Lavalley et al. 1992), which implements the methods presented in Isobe et al. (1986).

\section{DATA AVAILABILITY}

Data available on request.

\section{REFERENCES}

Aalto S., Booth R. S., Black J. H., Johansson L. E. B., 1995, A\&A, 300,369

Alam S. et al., 2015, ApJS, 219, 12

Allison J. R., Curran S. J., Sadler E. M., Reeves S. N., 2013, MNRAS, 430, 157

Allison J. R. et al., 2019, MNRAS, 482, 2934

Allison J. R. et al., 2017, MNRAS, 465, 4450

Allison J. R. et al., 2020, MNRAS, 494, 3627

Balashev S. A., Klimenko V. V., Ivanchik A. V., Varshalovich D. A., Petitjean P., Noterdaeme P., 2014, MNRAS, 440, 225

Borthakur S., Tripp T. M., Yun M. S., Momjian E., Meiring J. D., Bowen D. V., York D. G., 2010, ApJ, 713, 131

Carilli C. L., Menten K. M., Reid M. J., Rupen M. P., Yun M. S., 1998, ApJ, 494, 175 
Carilli C. L., Perlman E. S., Stocke J. T., 1992, ApJ, 400, L13

Carilli C. L., Rupen M. P., Yanny B., 1993, ApJ, 412, L59

Chengalur J. N., de Bruyn A. G., Narasimha D., 1999, A\&A, 343, L79

Combes F., Wiklind T., 1997, Academie des Sciences Paris Comptes Rendus Serie B Sciences Physiques, 5, 297

Curran S., Allison J. R., Whiting M. T., Sadler E. M., Combes F., Pracy M. B., Bignell C., Athreya R., 2016, MNRAS, 457, 3666

Curran S., Whiting M. T., Allison J. R., Tanna A., Sadler E. M., Athreya R., 2017, MNRAS, 467, 4514

Curran S. J., 2012, ApJ, 748, L18

Curran S. J., 2021, MNRAS, 506, 1548

Curran S. J., Allison J. R., Glowacki M., Whiting M. T., Sadler E. M., 2013, MNRAS, 431, 3408

Curran S. J., Darling J. K., Bolatto A. D., Whiting M. T., Bignell C., Webb J. K., 2007, MNRAS, 382, L11

Curran S. J., Hunstead R. W., Johnston H. M., Whiting M. T., Sadler E. M., Allison J. R., Athreya R., 2019, MNRAS, 484, 1182

Curran S. J., Kanekar N., Darling J. K., 2004, Science with the Square Kilometer Array, New Astronomy Reviews 48, Carilli C. L., Rawlings S., eds., Elsevier, Amsterdam, pp. 1095-1105

Curran S. J., Moss J. P., 2019, A\&A, 629, A56

Curran S. J., Moss J. P., Perrott Y. C., 2021, MNRAS, 503, 2639

Curran S. J. et al., 2011a, MNRAS, 416, 2143

Curran S. J. et al., 2011b, MNRAS, 413, 1165

Curran S. J., Whiting M. T., Murphy M. T., Webb J. K., Longmore S. N., Pihlström Y. M., Athreya R., Blake C., 2006, MNRAS, 371,431

Curran S. J., Whiting M. T., Wiklind T., Webb J. K., Murphy

M. T., Purcell C. R., 2008, MNRAS, 391, 765

Darling J. K., 2003, PhRvL, 91, 011301

Drinkwater M. J., Combes F., Wiklind T., 1996, A\&A, 312, 771

Drinkwater M. J., Webb J. K., Barrow J. D., Flambaum V. V., 1998, MNRAS, 295, 457

Dutta R., Raghunathan S., Gupta N., Joshi R., 2020, MNRAS, 491, 838

Dutta R., Srianand R., Gupta1 N., Momjian E., Noterdaeme P., Petitjean P., Rahmani H., 2017, MNRAS, 465, 588

Elíasdóttir Á., Hjorth J., Toft S., Burud I., Paraficz D., 2006, ApJS, 166,443

Falco E. E., Lehár J., Shapiro I. I., 1997, AJ, 113, 540

Feigelson E. D., Nelson P. I., 1985, ApJ, 293, 192

Grasha K., Darling J., Leroy A. K., Bolatto A. D., 2020, MNRAS, 498, 883

Grasha K., Darling J. K., Bolatto, A. D. Leroy A., Stocke J., 2019, ApJS, 245, 3

Gupta N. et al., 2021a, ApJ, 907, 11

Gupta N., Momjian E., Srianand R., Petitjean P., Noterdaeme P., Gyanchandani D., Sharma R., Kulkarni S., 2018a, ApJ, 860, L22 Gupta N., Srianand R., Bowen D. V., York D. G., Wadadekar Y., 2010, MNRAS, 408, 849

Gupta N. et al., 2018b, MNRAS, 476, 2432

Gupta N., Srianand R., Noterdaeme P., Petitjean P., Muzahid S., 2013, A\&A, 558, A84

Gupta N., Srianand R., Petitjean P., Noterdaeme P., Saikia D. J., 2009, MNRAS, 398, 201

Gupta N. et al., 2021b, ApJ, 255, 28

Henkel C., Jethava N., Kraus A., Menten K. M., Carilli C. L., Grasshoff M., Lubowich D., Reid M. J., 2005, A\&A, 440, 893

Isobe T., Feigelson E., Nelson P., 1986, ApJ, 306, 490

Israel F. P., 1988, in Millimetre and Submillimetre Astronomy,
Wolstencroft R. D., Burton W. B., eds., Kluwer, Dordrecht, pp. 281-305

Kanekar N., Briggs F. H., 2003, A\&A, 412, L29

Kanekar N. et al., 2005, PhRvL, 95, 261301

Kanekar N., Chengalur J. N., 2002, A\&A, 381, L73

Kanekar N., Chengalur J. N., de Bruyn A. G., Narasimha D., 2003, MNRAS, 345, L7

Kaplan E. L., Meier P., 1958, J. Amer. Statist. Assoc., 53, 457

Lavalley M. P., Isobe T., Feigelson E. D., 1992, in BAAS, Vol. 24, pp. $839-840$

Levshakov S. A., Varshalovich D. A., 1985, MNRAS, 212, 517

Maccagni F. M., Morganti R., Oosterloo T. A., Geréb K., Maddox N., 2017, A\&A, 604, A43

Moore C. B., Carilli C. L., Menten K. M., 1999, ApJ, 510, L87

Muller S. et al., 2013, A\&A, 551, 109

Muller S. et al., 2011, A\&A, 535, 103

Muller S., Jaswanth S., Horellou C., Martí-Vidal I., 2020, A\&A, 641, L2

Muller S. et al., 2016, A\&A, 595, A128

Muller S. et al., 2017, A\&A, 606, A109

Murphy M. T., Curran S. J., Webb J. K., 2003a, MNRAS, 342, 830

Murphy M. T., Webb J. K., Flambaum V. V., Curran S. J., 2003b, Astrophys. Space Sci., 283, 577

Noterdaeme P., Srianand R., Rahmani H., Petitjean P., Pâris I., Ledoux C., Gupta N., López S., 2015, A\&A, 577, A24

O’Dea C. P., Gallimore J., Stanghellini C., Baum S. A., Jackson J. M., 2005, AJ, 129, 610

Orienti M., Morganti R., Dallacasa D., 2006, A\&A, 457, 531

Skrutskie M. F. et al., 2006, AJ, 131, 1163

Srianand R., Gupta N., Momjian E., Vivek M., 2015, MNRAS, 451, 917

Tanna A., Curran S. J., Whiting M. T., Webb J. K., Bignell C., 2013, ApJ, 772, L25

Tercero B., Cernicharo J., Cuadrado S., de Vicente P., Guélin M., 2020, A\&A, 636, L7

Tonry J. L., Kochanek C. S., 1999, AJ, 117, 2034

Vermeulen R. C. et al., 2003, A\&A, 404, 861

Wallström S. H. J., Muller S., Roueff E., Le Gal R., Black J. H., Gérin M., 2019, A\&A, 629, A128

Webb J. K., King J. A., Murphy M. T., Flambaum V. V., Carswell R. F., Bainbridge M. B., 2011, PhRvL, 107, 191101

Wiklind T., Combes F., 1994, A\&A, 286, L9

Wiklind T., Combes F., 1995, A\&A, 299, 382

Wiklind T., Combes F., 1996a, Nat, 379, 139

Wiklind T., Combes F., 1996b, A\&A, 315, 86

Wiklind T., Combes F., 1997, A\&A, 328, 48

Wiklind T., Combes F., 1998, ApJ, 500, 129

Wiklind T., Combes F., 2001, in Gravitational Lensing, Brainerd T. G., Kochanek C. S., eds., ASP Conf. Ser. 237, San Francisco, p. 155

Wiklind T., Combes F., Kanekar N., 2018, ApJ, 864, 73

Wright E. L. et al., 2010, AJ, 140, 1868

Zheng Z., Li D., Sadler E. M., Allison J. R., Tang N., 2020, MNRAS, 499, 3085

Zwaan M. A., Prochaska J. X., 2006, ApJ, 643, 675 